\title{
Distributed Uplink Base Station Cooperation for Optimal SIR Assignment
}

\author{
Yosia Hadisusanto, Lars Thiele and Volker Jungnickel \\ Fraunhofer German-Sino Lab Mobile Communications (MCI) \\ Einsteinufer 37, D-10587 Berlin (Germany) \\ \{yosia.hadisusanto, thiele, jungnickel\}@ hhi.fraunhofer.de
}

\begin{abstract}
Base station cooperation is considered as a promising approach to increase the quality of service $(\mathrm{QoS})$ in uplink. Base stations are connected via high-capacity backhaul links, which makes it possible to perform joint detection through information sharing among several base stations. Joint detection broadens the feasible signal to interference ratio (SIR) region, in which any SIR assignment for all users can be concurrently supported. Thus it provides a higher system throughput. We characterized the Pareto-optimal boundary of the feasible SIR region of power control with base station cooperation by utilizing the property of concave interference function. We suggest a distributed power control algorithm that exploits the broader feasible SIR region and optimizes the SIR assignment. Depending on the feasibility of the network to support cooperation, we analyze 3 degrees of cooperation: no cooperation, limited cooperation, and full cooperation.
\end{abstract}

\section{INTRODUCTION}

In modern mobile communications systems, the network has to support multiple mobile stations simultaneously. Due to aggressive frequency reuse where several users are allocated simultaneously at the same frequency- and time resource, power control has a very important role in uplink. Without power control, cell-edge users would be disadvantaged by users located closer to the base stations, since the interference coming from the closer mobile stations overwhelm the signal of the cell-edge users. The quality of receive signal in base station depends on the ratio of signal to interference plus noise (SINR). The use of high power levels leads to a significant reduction in network performance, since the transmission power by one user is considered as interference from other users. Thus power control is important to control the multiuser interference.

The conventional distributed power control algorithm targets to minimize the overall transmit power while maintaining certain fixed SIR requirement (see [1] and references therein). The algorithm was motivated for wireless voice networks, namely to satisfy the minimum SIR requirement. However, in wireless data network the operator would like to support variable SIR assignment, where higher SIR provides a user with higher data rate, while smaller SIR can still support lower data rate applications. Due to multi-user interference, unfortunately not all SIR vectors can be supported. The feasible SIR region is defined as the region, in which any SIR assignment for all users can be concurrently supported. The SIR assignment can not go beyond the feasible SIR region. Thus it is important to find a distributed algorithm to assign SIRs that belong to the feasible SIR region and yet optimal. A distributed power control algorithm, which achieves the optimal SIR assignment, has been recently found [2].

In future wireless networks, base stations are connected via high-capacity backhaul links, which makes it possible to perform joint detection among the distributed antenna arrays through information sharing between several base stations. This additional degrees of freedom broadens the feasible SIR region and provides a higher system throughput. Base station cooperation for uplink wireless networks has been considered in [3]. However, as their objective is to minimize total transmit power with fixed SIR assignment, the algorithm doesn't fully exploit the knowledge of the broader feasible SIR region due to base station cooperation. This paper extends the distributed power control algorithm in [2] with the aim to exploit the broader SIR region and to approach an optimal SIR assignment for uplink base station cooperation. While the feasible SIR region in [2] has a log-convexity property, which allows convex optimization problem formulation and ensuring global optimal solution, unfortunately the feasible SIR region for base station cooperation is generally not log-convex. The optimal SIR assignment solution must lie on the Pareto-optimal boundary of the feasible SIR region to achieve the highest possible efficiency. Fortunately, the boundary of the feasible SIR region for base station cooperation can be characterized by using the property of the concave interference function [4]. Therefore the optimization problem over the whole feasible region can be transformed to the optimization along the Pareto-optimal boundary. Depending on the feasibility of the network to support cooperation, we analyze 3 degrees of cooperation: no cooperation, limited cooperation, and full cooperation.

\section{System Model And Problem Formulation}

Consider the uplink of a wireless cellular network with $M$ mobile stations, each equipped with 1 transmit antenna, sending independent information to $N$ base stations, where each base station is equipped with $K$ receive antennas. Assuming the cellular network is being connected via a high-speed optical fiber backbone such that message passing between several base stations is possible and a signal can be jointly received and coherently combined by several base stations. The signal transmitted by mobile station $i$ is detected by a set of base stations $\boldsymbol{\pi}_{i}$. The cardinality $\left|\boldsymbol{\pi}_{i}\right|$ is the number of base 


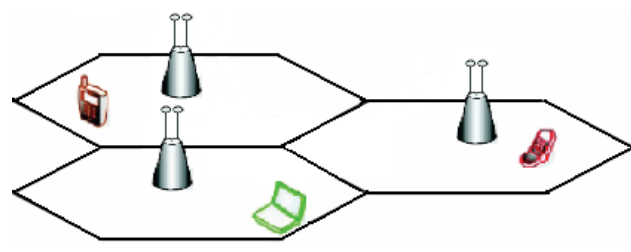

Fig. 1. Uplink system model.

stations which serve user $i$. We assume that the cardinality $\left|\boldsymbol{\pi}_{i}\right|$ depends by the feasibility of the network to support cooperation (i.e. no cooperation, limited cooperation, or full cooperation). Denote $\mathbf{h}_{\boldsymbol{\pi}_{i} i}$ as the $\left|\boldsymbol{\pi}_{i}\right| K \times 1$ complex valued channel vector from mobile station $i$ to its serving cooperative base stations $\boldsymbol{\pi}_{i}$. These channel vectors are estimated at base stations using pilot signals transmitted by mobile stations. Let $x_{i}$ be the information signal sent by mobile $i$. The receiver, which is the set of cooperative base stations $\boldsymbol{\pi}_{i}$, employs a linear joint beamforming vector $\mathbf{w}_{i} \in \mathcal{C}^{\left|\boldsymbol{\pi}_{i}\right| K \times 1}$, with $\left\|\mathbf{w}_{i}\right\|=1$, resulting in a received signal for mobile station $i$ given by

$$
\mathbf{w}_{i}^{H} \mathbf{y}_{\boldsymbol{\pi}_{i} i}=\underbrace{\left(\mathbf{w}_{i}^{H} \mathbf{h}_{\boldsymbol{\pi}_{i} i}\right) x_{i}}_{\text {Useful signal for MS } i}+\underbrace{\sum_{j \neq i}\left(\mathbf{w}_{i}^{H} \mathbf{h}_{\boldsymbol{\pi}_{i} j}\right) x_{j}}_{\text {Interference from another MS }}+\underbrace{\mathbf{w}_{i}^{H} \mathbf{z}_{\boldsymbol{\pi}_{i}}}_{\text {Noise }},
$$

where $\mathbf{z}_{\boldsymbol{\pi}_{i}}$ is a vector of dimension $\left|\boldsymbol{\pi}_{i}\right| K \times 1$, which contains the additive white Gaussian noise (AWGN) component with variance $\sigma^{2}$, i.e. $E\left[\mathbf{z}_{\boldsymbol{\pi}_{i}} \mathbf{z}_{\boldsymbol{\pi}_{i}}^{H}\right]=\sigma^{2} \mathbf{I}$. Let $E\left\{\left|x_{i}\right|^{2}\right\}=p_{i}$ be the transmit power from mobile $i$. The transmit power from all users are collected in a power allocation vector, $\mathbf{p}=\left[p_{1}, \ldots, p_{M}\right]^{T}$. The instantaneous SIR, $\gamma_{i}$, for link $i$ is $\gamma_{i}=\frac{p_{i}\left|\mathbf{w}_{i}^{H} \mathbf{h}_{\pi_{i} i}\right|^{2}}{\sum_{j \neq i} p_{j}\left|\mathbf{w}_{i}^{H} \mathbf{h}_{\pi_{i} j}\right|^{2}+\sigma^{2}}$. The interference coupling matrix is defined as in [5]

$$
[\mathbf{V}(\mathbf{W}, \boldsymbol{\Pi})]_{i j}= \begin{cases}\frac{\mathbf{w}_{i}^{H} \mathbf{R}_{\pi_{i} j} \mathbf{w}_{i}}{\mathbf{w}_{i}^{H} \mathbf{R}_{\pi_{i} i} \mathbf{w}_{i}}, & \text { if } i \neq j \\ 0, & \text { if } i=j\end{cases}
$$

where the matrix $\mathbf{R}_{\boldsymbol{\pi}_{i j}}=E\left[\mathbf{h}_{\boldsymbol{\pi}_{i} j} \mathbf{h}_{\boldsymbol{\pi}_{i j}}^{H}\right]$ is the spatial covariance matrix of the vector channel $\mathbf{h}_{\boldsymbol{\pi}_{i} j}$. The interference coupling matrix, $\mathbf{V}(\mathbf{W}, \boldsymbol{\Pi})$, contains interference coefficients, which determine in which way the users' transmit signals are affected by multi-user interference. It depends on the receive beamforming vectors $\mathbf{w}_{1}, \ldots, \mathbf{w}_{M}$, which are collected in matrix $\mathbf{W}$, and the cooperative base stations assignment vectors $\boldsymbol{\pi}_{1}, \ldots, \boldsymbol{\pi}_{M}$, which are collected in matrix $\Pi$. The interference experienced by the $i$ th user can be modeled by an interference function, which is defined as

$$
I_{i}(\mathbf{p}, \mathbf{W}, \boldsymbol{\Pi})=[\mathbf{V}(\mathbf{W}, \boldsymbol{\Pi}) \mathbf{p}]_{i}+c_{i}, \quad i=1, \ldots, M,
$$

with $c_{i}=\frac{\sigma^{2}}{\mathbf{w}_{i}^{H} \mathbf{R}_{\pi_{i} i} \mathbf{w}_{i}}$. Note that if we have a constant $\mathbf{W}$ and a constant $\Pi$, the interference function $I_{i}(\mathbf{p})$ is linear. We can stack the interference function in a vector, so that

$$
I(\mathbf{p}, \mathbf{W}, \boldsymbol{\Pi})=\mathbf{V}(\mathbf{W}, \boldsymbol{\Pi}) \mathbf{p}+\mathbf{c} .
$$

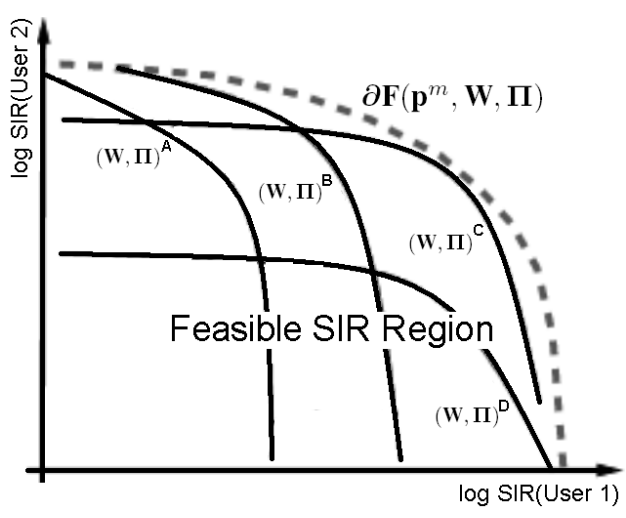

Fig. 2. The feasible SIR region is the union of regions associated with all possible combinations of $(\mathbf{W}, \boldsymbol{\Pi})$. Each $(\mathbf{W}, \boldsymbol{\Pi})^{*}$ stands for a fixed choice of beamformer coefficients and cooperative BS assignment sets. By varying the power vector $\mathbf{p}$, we get the associated trade-off curve. $\partial \mathbf{F}\left(\mathbf{p}^{m}, \mathbf{W}, \mathbf{\Pi}\right)$ is the Pareto-boundary of the feasible SIR region [5].

Let $\gamma_{i}$ be the SIR achieved by link $i$ with $\gamma_{i}=$ $p_{i} / I_{i}(\mathbf{p}, \mathbf{W}, \boldsymbol{\Pi})$ and define an SIR assignment matrix $\boldsymbol{\Gamma}(\gamma)=$ $\operatorname{diag}\left(\gamma_{1}, \ldots, \gamma_{M}\right)$, then

$$
\mathbf{p}=\boldsymbol{\Gamma}(\gamma) I(\mathbf{p}, \mathbf{W}, \boldsymbol{\Pi})
$$

The power allocation equation can be formulated as

$$
\mathbf{p}=(\mathbf{I}-\boldsymbol{\Gamma}(\gamma) \mathbf{V}(\mathbf{W}, \boldsymbol{\Pi}))^{-1} \boldsymbol{\Gamma}(\gamma) \mathbf{c} .
$$

Since the power allocation vector must be non-negative, i.e. $\mathbf{p} \succeq 0$, it is required that $(\mathbf{I}-\boldsymbol{\Gamma}(\boldsymbol{\gamma}) \mathbf{V}(\mathbf{W}, \boldsymbol{\Pi}))^{-1}$ to be also non-negative. Thus not all SIR assignments $\gamma$ are achievable. A clever selection of each user's cooperative base stations assignment and beamforming vector will cause the interference coupling matrix $\mathbf{V}(\mathbf{W}, \boldsymbol{\Pi})$ to have a smaller value, thus can support a higher SIRs. By the Perron-Frobenius theorem, an SIR assignments $\boldsymbol{\Gamma}(\boldsymbol{\gamma})$ is feasible if and only if $\rho(\boldsymbol{\Gamma}(\boldsymbol{\gamma}) \mathbf{V}(\mathbf{W}, \boldsymbol{\Pi}))<1$, where $\rho($.$) is a spectral radius$ function [6]. Thus the unconstrained feasible SIR region $\gamma$ is $\mathbf{B}(\mathbf{W}, \boldsymbol{\Pi})=\{\boldsymbol{\gamma} \succeq 0: \rho(\boldsymbol{\Gamma}(\boldsymbol{\gamma}) \mathbf{V}(\mathbf{W}, \boldsymbol{\Pi}))<1\}$. If the interference coupling matrix $\mathbf{V}$ doesn't depend on other variables, it is known that the logarithm of the feasible SIR region is convex [7]. However since now the interference coupling matrix $\mathbf{V}(\mathbf{W}, \boldsymbol{\Pi})$ depends on the joint beamforming strategy $\mathbf{W}$ and cooperative base stations assignment strategy $\boldsymbol{\Pi}$, then the feasible SIR region $\mathbf{B}(\mathbf{W}, \boldsymbol{\Pi})$ becomes the union of regions associated with all possible combinations of $\mathbf{W}$ and $\Pi$ I. Generally the union of convex regions is not a convex region. Taking the maximum transmit power constraint $\mathbf{p}^{m}$ into account, the power allocation must satisfy $\mathbf{p} \preceq \mathbf{p}^{m}$. The power-constrained feasible SIR region is defined as

$$
\mathbf{F}\left(\mathbf{p}^{m}, \mathbf{W}, \boldsymbol{\Pi}\right)=\left\{\boldsymbol{\gamma} \in \mathbf{B}(\mathbf{W}, \Pi) \mid \mathbf{p}(\gamma) \preceq \mathbf{p}^{m}\right\}
$$

The SIR optimization problem for uplink base station cooperation over non-convex feasible SIR region, $\mathbf{F}\left(\mathbf{p}^{m}, \mathbf{W}, \mathbf{\Pi}\right)$ can be formulated as a Network Utility Maximization (NUM) 
problem

$$
\begin{array}{rll}
\max . & : & \sum_{i=1}^{M} U_{i}\left(\beta\left(\gamma_{i}\right)\right) \\
\text { s.t. } & : \quad \gamma \in \mathbf{F}\left(\mathbf{p}^{m}, \mathbf{W}, \mathbf{\Pi}\right) \\
& \mathbf{p} \succeq \mathbf{0} \\
\text { variables } & : \quad \mathbf{p}, \mathbf{W}, \boldsymbol{\Pi}, \boldsymbol{\gamma} .
\end{array}
$$

A class of utility functions is introduced in [8], where different optimization goals can be achieved by varying the parameter $\alpha \geq 1$

$$
U_{i}\left(\beta_{i}\right)=\left\{\begin{array}{ll}
\log \left(\beta_{i}\right) & \text { if } \alpha=1, \\
\frac{\beta_{i}^{1-\alpha}}{1-\alpha} & \text { if } \alpha>1 .
\end{array},\right.
$$

and we define the user throughput $\beta_{i}=\log _{2}\left(1+\gamma_{i}\right)$ as the QoS metric. In this paper we consider proportional fairness utility with $\alpha=1$ to create a balance between maximizing total wireless network throughput while at the same time allowing all users at least a minimal level of service.

\section{OPTIMIZATION USING CONCAVE INTERFERENCE FUNCTIONS}

The assignment of $\boldsymbol{\pi}_{i}$ and $\mathbf{w}_{i}$ can be optimized independently for each user, since it doesn't influence the interference perceived by other users, thus we get a concave interference function [4]

$$
\begin{array}{ll}
I_{i}^{\text {opt }} & (\mathbf{p}) \\
= & \min _{\boldsymbol{\pi}_{i}: 1 \leq\left|\boldsymbol{\pi}_{i}\right| \leq N}\left(\min _{\mathbf{w}_{i}:\left\|\mathbf{w}_{i}\right\| \mid=1}[\mathbf{V}(\mathbf{W}, \boldsymbol{\Pi}) \mathbf{p}]_{i}+\frac{\sigma^{2}}{\mathbf{w}_{i}^{H} \mathbf{R}_{\boldsymbol{\pi}_{i} i \mathbf{w}_{i}}}\right) \\
= & \min _{\boldsymbol{\pi}_{i}: 1 \leq\left|\boldsymbol{\pi}_{i}\right| \leq N}\left(\min _{\mathbf{w}_{i}:\left\|\mathbf{w}_{i}\right\|=1} \mathbf{V}_{i}\left(\mathbf{w}_{i}, \boldsymbol{\pi}_{i}\right) \mathbf{p}+\frac{\sigma^{2}}{\mathbf{w}_{i}^{H} \mathbf{R}_{\boldsymbol{\pi}_{i} i} \mathbf{w}_{i}}\right) \\
= & \min _{\boldsymbol{\pi}_{i}: 1 \leq\left|\boldsymbol{\pi}_{i}\right| \leq N} \frac{1}{\mathbf{h}_{\boldsymbol{\pi}_{i} i}^{H}\left(\sigma^{2} \mathbf{I}+\sum_{j \neq i} p_{j} \mathbf{h}_{\boldsymbol{\pi}_{i} j} \mathbf{h}_{\boldsymbol{\pi}_{i} j}^{H}\right)^{-1} \mathbf{h}_{\boldsymbol{\pi}_{i} i}} .
\end{array}
$$

Concave interference function belongs to the standard interference function [1], which fulfills the following axioms:

- A1 Positivity $I_{i}(\mathbf{p})>0$.

- A2 Monotonicity if $\mathbf{p} \geq \mathbf{p}^{\prime}$, then $I_{i}(\mathbf{p}) \geq I_{i}\left(\mathbf{p}^{\prime}\right)$.

- A3 Scalability $\alpha I_{i}(\mathbf{p}) \geq I_{i}(\alpha \mathbf{p})$ if $\alpha>1$.

The concave interference function, $I_{i}^{\text {opt }}(\mathbf{p})$, attains the minimum interference among all choices of cooperative base stations assignment and beamforming vector at power allocation p. The corresponding optimal linear beamformer for $I_{i}^{\text {opt }}(\mathbf{p})$ is the Minimum Mean Square Error (MMSE) beamformer [9] [10]:

$$
\mathbf{w}_{i}^{o p t}(\mathbf{p})=\left(\sum_{j \neq i} p_{j} \mathbf{h}_{\boldsymbol{\pi}_{i j}} \mathbf{h}_{\boldsymbol{\pi}_{i j}}^{H}+\sigma^{2} \mathbf{I}\right)^{-1} \mathbf{h}_{\boldsymbol{\pi}_{i} i} .
$$

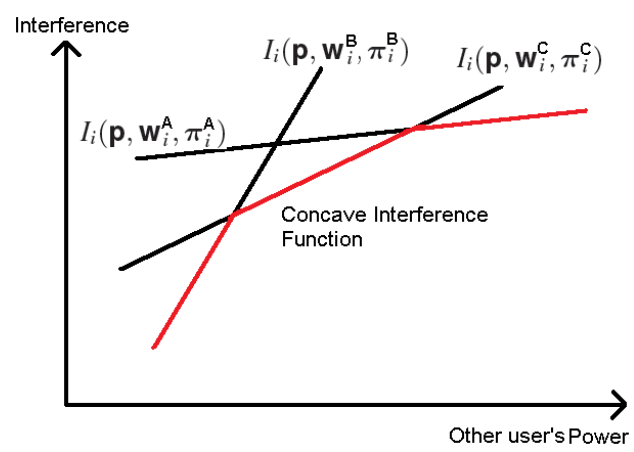

Fig. 3. Concave interference function attains the minimum interference among all choices of cooperative BS assignment and beamforming strategy.

The optimal choice of cooperative base stations assignment corresponds to $I_{i}^{o p t}(\mathbf{p})$ is

$$
\begin{array}{ll}
\boldsymbol{\pi}_{i}^{\text {opt }} & (\mathbf{p}) \\
= & \underset{\boldsymbol{\pi}_{i}: 1 \leq\left|\boldsymbol{\pi}_{i}\right| \leq N}{\operatorname{argmin}}\left(\left[\mathbf{V}\left(\mathbf{W}^{\text {opt }}, \boldsymbol{\pi}\right) \mathbf{p}\right]_{i}+\frac{\sigma^{2}}{\mathbf{w}_{i}^{\text {opt }} \mathbf{R}_{\boldsymbol{\pi}_{i} i} \mathbf{w}_{i}^{\text {opt }}}\right) .
\end{array}
$$

Based on the cardinality of the cooperative base stations assignment $\left|\boldsymbol{\pi}_{i}\right|$, which is defined by the feasibility of the network to support cooperation, we can define 3 degrees of cooperation:

\section{1) No Cooperation.}

If $\left|\boldsymbol{\pi}_{i}\right|=1 \quad \forall i$, we assume that there is no cooperation between base stations. In this case, user $i$ is assigned to the base station at which its interference function is minimized.

$$
\begin{array}{ll}
I_{i}^{\text {opt }} & (\mathbf{p}) \\
= & \min _{\boldsymbol{\pi}_{i}:\left|\boldsymbol{\pi}_{i}\right|=1} \frac{1}{\mathbf{h}_{\boldsymbol{\pi}_{i} i}^{H}\left(\sigma^{2} \mathbf{I}+\sum_{j \neq i} p_{j} \mathbf{h}_{\boldsymbol{\pi}_{i} j} \mathbf{h}_{\boldsymbol{\pi}_{i j}}^{H}\right)^{-1} \mathbf{h}_{\boldsymbol{\pi}_{i} i}}
\end{array}
$$

\section{2) Full Cooperation.}

The transmitted signal of user $i$ is jointly detected at $\left|\boldsymbol{\pi}_{i}\right|=N$ cooperative base stations. This case achieves the maximum performance from these 3 degrees of cooperation, since the interference function is totally minimized by joint detection from all base stations.

$$
I_{i}^{o p t}(\mathbf{p})=\frac{1}{\mathbf{h}_{\boldsymbol{\pi}_{i} i}^{H}\left(\sigma^{2} \mathbf{I}+\sum_{j \neq i} p_{j} \mathbf{h}_{\boldsymbol{\pi}_{i} j} \mathbf{h}_{\boldsymbol{\pi}_{i j}}^{H}\right)^{-1} \mathbf{h}_{\boldsymbol{\pi}_{i} i}}
$$

\section{3) Limited Cooperation.}

The limited cooperation case is considered, in which the received signal of user $i$ is jointly detected at $d_{i}$ cooperative base stations with $1<d_{i}<N$. This criteria is more feasible than full cooperation case, since only limited number of base stations need to perform joint detection. However there are $\left(\begin{array}{l}N \\ d_{i}\end{array}\right)$ possibilities to find the optimal combination of $d_{i}$ cooperative base stations 
from the overall $N$ base stations which minimizes the interference function.

$$
\begin{array}{ll}
I_{i}^{o p t} & (\mathbf{p}) \\
= & \min _{\boldsymbol{\pi}_{i}:\left|\boldsymbol{\pi}_{i}\right|=d_{i}} \frac{1}{\mathbf{h}_{\boldsymbol{\pi}_{i} i}^{H}\left(\sigma^{2} \mathbf{I}+\sum_{j \neq i} p_{j} \mathbf{h}_{\boldsymbol{\pi}_{i} j} \mathbf{h}_{\boldsymbol{\pi}_{i} j}^{H}\right)^{-1} \mathbf{h}_{\boldsymbol{\pi}_{i} i}}
\end{array}
$$

The optimal SIR assignment solution must lie on the Paretoboundary of the feasible SIR region to achieve the highest possible efficiency. Using the property of concave interference function we can characterize the Pareto-boundary of the feasible SIR region.

Theorem 1: Pareto-Boundary Characterization of the Feasible SIR Region of BS Cooperation

Let $\boldsymbol{\partial F}\left(\mathbf{p}^{m}, \mathbf{W}, \boldsymbol{\Pi}\right)$ be the Pareto-boundary of the feasible SIR region. The interference vector $I(\mathbf{p}, \mathbf{W}, \boldsymbol{\Pi})$ on $\boldsymbol{\partial \mathbf { F }}\left(\mathbf{p}^{m}, \mathbf{W}, \boldsymbol{\Pi}\right)$ must be the concave interference function $I^{o p t}(\hat{\mathbf{p}})$ with at least one user transmits with full power: $\hat{\mathbf{p}} \in\left\{\mathbf{p} \preceq \mathbf{p}^{m} \mid \exists i: p_{i}=\right.$ $\left.p_{i}^{m}\right\}$.

Proof: The power allocation vector on the Pareto-optimal boundary must be in the set $\left\{\mathbf{p} \preceq \mathbf{p}^{m} \mid \exists i: p_{i}=p_{i}^{m}\right\}$, which is proved in [2]. Suppose the tupple $\left(\hat{\mathbf{p}}, \mathbf{W}^{\prime}, \boldsymbol{\Pi}^{\prime}\right)$ is the power-, beamformer-, and cooperative BSs assignment vector on Pareto-boundary, so that $\gamma^{\prime} \in \partial \mathbf{F}\left(\mathbf{p}^{m}, \mathbf{W}, \mathbf{\Pi}\right)$ with interference vector $I^{\prime}(\hat{\mathbf{p}})$. Assume that the pair $\left(\mathbf{W}^{\prime}, \boldsymbol{\Pi}^{\prime}\right) \neq$ $\left(\mathbf{W}^{o p t}, \boldsymbol{\Pi}^{o p t}\right)$. From equation (10) we know that $I_{i}^{o p t}(\hat{\mathbf{p}}) \leq$ $I^{\prime}(\hat{\mathbf{p}})$ for each user, which means that for the same user's transmit power $\hat{p}_{i}$ then $\gamma_{i}^{\text {opt }} \geq \gamma_{i}^{\prime}$ for each user. Thus proving the claim by contradiction.

Thus by utilizing the concave interference function $I^{\text {opt }}(\mathbf{p})$ from equation (10), the optimization problem (8), which is over the whole feasible SIR region $\mathbf{F}\left(\mathbf{p}^{m}, \mathbf{W}, \boldsymbol{\Pi}\right)$, can be transformed to the optimization along the Pareto-optimal boundary $\partial \mathbf{F}\left(\mathbf{p}^{m}, \mathbf{W}, \boldsymbol{\Pi}\right)$ since the optimal SIR assignment must lie on the Pareto-boundary

$$
\begin{aligned}
\max . & : \sum_{i=1}^{M} U_{i}\left(\gamma_{i}\right) \\
\text { s.t. } \quad: \quad & p_{i} \geq \gamma_{i} I_{i}^{\text {opt }}(\mathbf{p}), \quad i=1,2, \ldots, M \\
& \mathbf{0} \preceq \mathbf{p} \preceq \mathbf{p}^{m} \\
\text { variables } \quad: \quad & \mathbf{p}, \mathbf{W}, \boldsymbol{\Pi}, \boldsymbol{\gamma} .
\end{aligned}
$$

To solve this optimization problem, we combine the ascentdirection algorithm from [2] and the concave interference function $I_{i}^{o p t}(\mathbf{p})$ to develop a greedy algorithm, which searches a local optimum solution on the Pareto boundary. Ascentdirection means that $\sum_{i=1}^{M} U_{i}\left(\gamma_{i}[t+1]\right) \geq \sum_{i=1}^{M} U_{i}\left(\gamma_{i}[t]\right)$, where $t$ is the iteration index. The power-interference representation is re-parameterized into the so-called load-spillage representation, so that the SIR is re-parameterized into a loadspillage term

$$
\gamma_{i}[t]=l_{i}[t] / s_{i}[t]
$$

Spillage $s_{i}$ represents the effect of interference emitted by mobile station $i$ transmission on other users in the network

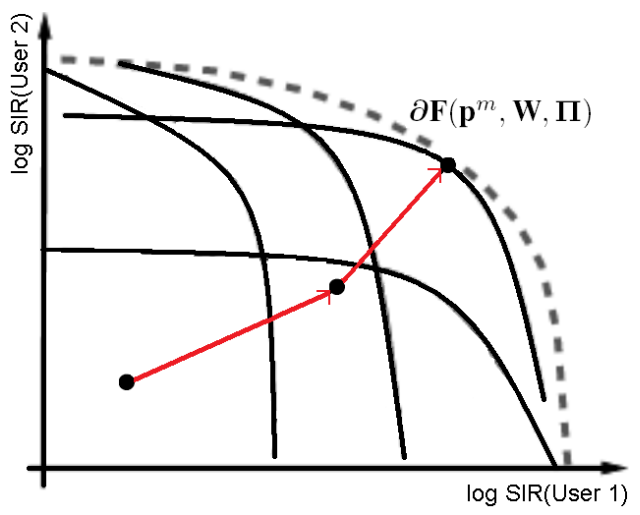

Fig. 4. Illustration of the algorithm. The algorithm starts with an initialization point and goes iteratively to the boundary of the feasible SIR region until local optimum SIR assignment is achieved.

weighted by other user loads $l_{j}$ plus the power price $\nu_{i}$.

$$
s_{i}[t]=\sum_{j}\left[\mathbf{V}\left(\mathbf{W}^{o p t}, \boldsymbol{\Pi}^{o p t}\right)[t]\right]_{j i} l_{j}[t]+\nu_{i}[t] .
$$

A mobile station with large spillage would easily carry over interference into another mobile stations and hence should be assigned a smaller SIR. Link $i$, with an $\operatorname{SIR} \gamma_{i}$, and responsible for spillage $s_{i}$, loads the network with $l_{i}=s_{i} \gamma_{i}$ in the sense that it is less tolerant by a factor of $l_{i}$ to interference from other users in the network. The power price $\nu_{i}$ for mobile station $i$ is updated depending upon the transmit power constraint $p_{i}^{m}$ and is determined through an iterative subgradient update to bring down the SIR assignment from unconstrained feasible SIR region $\mathbf{B}(\mathbf{W}, \boldsymbol{\Pi})$ to power constrained feasible SIR region $\mathbf{F}\left(\mathbf{p}^{m}, \mathbf{W}, \boldsymbol{\Pi}\right)$.

$$
\nu_{i}[t+1]=\left[\nu_{i}[t]+\delta_{\nu o}\left(p_{i}[t]-p_{i}^{m}\right)\right]^{+},
$$

where $\delta_{\nu o}>0$, is a sufficiently-small step-size [11]. The term $[z]^{+}=\max \{z, 0\}$ denotes the projection onto the nonnegative orthant. The larger the load factor user $i$ has, the more immune is user $i$ against interference from other users. Therefore, the load factor is chosen in greedy manner, where in each step the search direction is towards the largest load each user can have by utilizing concave interference function

$$
\begin{aligned}
l_{i}[t+1] & =l_{i}[t]+\delta_{l} \triangle l_{i}[t+1] \\
\triangle l_{i}[t+1] & =\frac{U_{i}^{\prime}\left(\gamma_{i}[t]\right) \gamma_{i}[t]}{I_{i}^{o p t}(\mathbf{p}[t+1])}-l_{i}[t],
\end{aligned}
$$

where $\delta_{l}>0$ is a sufficiently small step-size for the load factor [11] and $U_{i}^{\prime}\left(\gamma_{i}\right)=\frac{1}{\ln 2} \frac{1}{1+\gamma_{i}} \frac{1}{\log _{2}\left(1+\gamma_{i}\right)}$. The ascent-direction update arrives on the Pareto-optimal boundary after it satisfies Theorem 1, namely as $\mathbf{p}[t] \in\left\{\mathbf{p} \preceq \mathbf{p}^{m} \mid \exists i: p_{i}=p_{i}^{m}\right\}$ and the current interference $I(\mathbf{p}[t], \mathbf{W}, \boldsymbol{\pi})=I^{\text {opt }}(\mathbf{p}[t])$. Then it slides along the Pareto-optimal boundary in ascent-direction and converges towards the local utility-optimal point.

For limited cooperation, $1<d_{i}<N$, however, the complexity to find an optimal cooperative base stations assignment to determine $I_{i}^{o p t}(\mathbf{p})$ is prohibitive due to $\left(\begin{array}{l}N \\ d_{i}\end{array}\right)$ possible combinations. Therefore for limited cooperation we propose a 
TABLE I

SIMULATION ASSUMPTIONS.

\begin{tabular}{l|r}
\hline $\begin{array}{l}\text { channel model } \\
\text { scenario } \\
f_{c}\end{array}$ & $\begin{array}{r}\text { 3GPP SCME } \\
\text { urban-macro }\end{array}$ \\
intersite distance & $2 \mathrm{GHz}$ \\
\hline $\begin{array}{l}\text { number of BS } \\
\text { BS height } \\
\text { number of receive antenna each }\end{array}$ & $300 \mathrm{~m}$ \\
\hline $\begin{array}{l}\text { number of MS in each cell } \\
\text { MS height } \\
\begin{array}{l}\text { number of transmit antenna each } \\
\text { transmit power constraint }\end{array}\end{array}$ \\
\hline
\end{tabular}

sub-optimal cooperation strategy, where the cooperative base stations assignment is chosen based on its link gain. Thus the interference function becomes

$$
I_{i}^{\text {subopt }}(\mathbf{p})=\frac{1}{\mathbf{h}_{\boldsymbol{\pi}_{i} i}^{H}\left(\sigma^{2} \mathbf{I}+\sum_{j \neq i} p_{j} \mathbf{h}_{\boldsymbol{\pi}_{i} j} \mathbf{h}_{\boldsymbol{\pi}_{i} j}^{H}\right)^{-1} \mathbf{h}_{\boldsymbol{\pi}_{i} i}},
$$

where $\boldsymbol{\pi}_{i}$ is a $d_{i}$ element set and its link gain has the maximal energy norm $\left\|\mathbf{h}_{\boldsymbol{\pi}_{i} i}\right\|_{2}$. The interference function $I_{i}^{\text {subopt }}(\mathbf{p})$ is still concave, since the interference function is minimized through the optimal beamforming vector in Eq. (11). The price that we pay for this simplification is the smaller feasible SIR region, since the interference coupling matrix depends only on beamforming $\mathbf{V}(\mathbf{W})$. It is important to note that under fullcooperation case, the performance of the optimal and suboptimal cooperative base stations assignment are the same, since each user is assigned to all cooperative base stations.

The distributed algorithm exploits the high-capacity backhaul links connecting the base stations for iteratively exchange information between them. After several iterations when the convergence is achieved, the cooperative base stations $\boldsymbol{\pi}_{i}$ communicates the optimal power to mobile station $i$.

\section{Simulation Results}

In order to evaluate the performance of uplink base station cooperation, we simulate the algorithm in system-level simulator by using the 3GPP Spatial Channel Model Extended (SCME) [12]. We consider frequency flat channels to analyze the performance. We use omnidirectional antenna array with two elements at the base station. Each cell is loaded with one user. Each mobile station is equipped with a single antenna. The maximum transmit power for each mobile station is 1 $\mathrm{mW}$. The simulation assumptions are given in the Table I. The geometric mean of the user throughput is used as a measure of the network QoS as in [2]. It is computed as the exponent of the mean proportional fairness utility over $\beta_{i}\left(\gamma_{i}\right)$

$$
\left[\prod_{i=1}^{M} \beta_{i}\left(\gamma_{i}\right)\right]^{1 / M}=\exp \left(\frac{1}{M} \sum_{i=1}^{M} \log \beta_{i}\left(\gamma_{i}\right)\right)
$$

Figure 5 shows the convergence behavior of the algorithm for different degrees of cooperation, namely no cooperation,

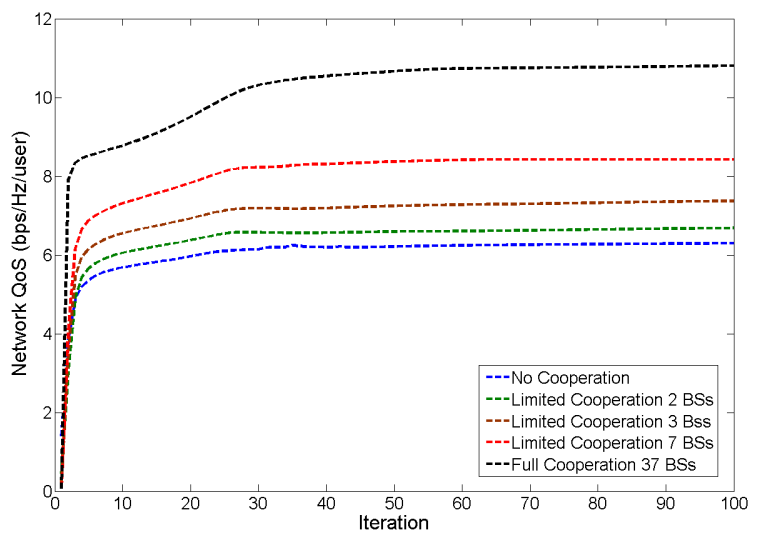

Fig. 5. Convergence behavior of the algorithm for different degrees of cooperation.

limited cooperation with 2,3 , and 7 cooperative base stations, and full cooperation. Due to ascent-direction updates of the algorithm, the QoS of the network increases with each iteration step until it reaches a convergence state. From Figure 5 we observe that with each addition of cooperative base station the QoS performance of the network increases. Figure 6 shows the cumulative distribution functions (CDFs) of user throughput. The CDFs are obtained using the maximization of the sum of proportional fairness utility function of 500 network instances. It can be seen that as the number of cooperative base station increases, the user throughput increases as well. We observe that almost half of the performance increase with full cooperation can be obtained when only 7 base stations with the strongest links are performing joint detection.

\section{Conclusions}

We have introduced the consideration of power control for uplink base station cooperation to achieve an optimal SIR assignment. We characterized the Pareto-optimal boundary of the feasible SIR region of power control with base station cooperation by utilizing the property of concave interference function. We developed a distributed greedy algorithm which achieves utility-optimal point on the Pareto-optimal boundary. It has been shown that base station cooperation broadens the feasible SIR region and provide a higher system throughput.

\section{REFERENCES}

[1] R. D. Yates, "A framework for uplink power control in cellular radio systems," IEEE Journal on Selected Areas in Communications, vol. 13, pp. 1341-1347, 1995.

[2] P. Hande, S. Rangan, M. Chiang, and X. Wu, "Distributed uplink power control for optimal sir assignment in cellular data networks," Networking, IEEE/ACM Transactions on, vol. 16, no. 6, pp. 1420-1433, Dec. 2008.

[3] S. Venkatesan, "Coordinating base stations for greater uplink spectral efficiency in a cellular network," in Personal, Indoor and Mobile Radio Communications, 2007. PIMRC 2007. IEEE 18th International Symposium on, Sept. 2007, pp. 1-5.

[4] H. Boche, M. Schubert, E. Jorswieck, and A. Sezgin, "A General Framework for Concave/Convex Interference Coordination Problems and Network Utility Optimization," in Proc. WSA, 2007. 


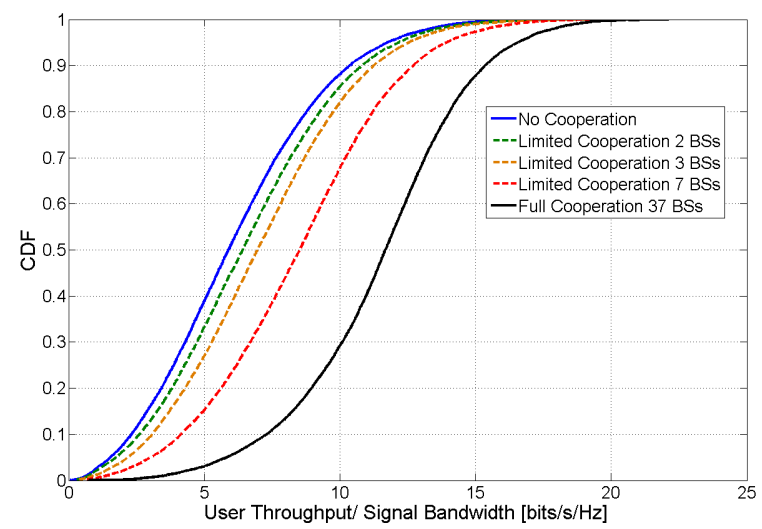

Fig. 6. CDFs of user throughput.

[5] M. Schubert and H. Boche, QoS-Based Resource Allocation and Transceiver Optimization, vol. 2 of Now the essence of knowledge, S. Verdu, Princeton University, foundations and trends in communications and information theory edition, 2005.

[6] J. Zander, "Performance of optimum transmitter power control in cellular radio systems," Vehicular Technology, IEEE Transactions on, vol. 41, no. 1, pp. 57-62, Feb 1992.

[7] C.W. Sung, "Log-convexity property of the feasible sir region in powercontrolled cellular systems," IEEE COMMUNICATIONS LETTERS, vol. 6, no. 6, pp. 248-249, 2002.

[8] J. Mo and J. Walrand, "Fair end-to-end window-based congestion control," IEEE/ACM Transactions on Networking, vol. 8, no. 5, pp. 556-567, 2000.

[9] R. A. Monzingo and T. W. Miller, "Introduction to adaptive arrays," 1980.

[10] J.H. Winters, "Optimum combining in digital mobile radio with cochannel interference," Vehicular Technology, IEEE Transactions on, vol. 33, no. 3, pp. 144-155, Aug 1984.

[11] S. Boyd, L. Xiao, and A. Mutapcic, "Subgradient methods," lecture notes of EE392o, Autumn Quarter 2003-2004.

[12] "Spatial channel model for multiple input multiple output (MIMO) simulations, TR 25.996 V7.0.0 (release 7)," Tech. Rep., 3rd generation partnership project (3GPP); technical specification group radio access network, July 2007. 